\title{
(6) OPEN ACCESS \\ Factors associated with low unit cohesion in Australian Defence Force members who deployed to the Middle East (2001-2009)
}

\author{
Jeeva Kanesarajah, ${ }^{1}$ M Waller, ${ }^{1}$ W Y Zheng, ${ }^{2}$ A J Dobson ${ }^{1}$
}

\begin{abstract}
- Additional material is published online only. To view please visit the journal online (http://dx.doi.org/10.1136/ jramc-2015-000484).

${ }^{1}$ School of Public Health, The University of Queensland, Brisbane, Australia

${ }^{2}$ School of Aviation, University of New South Wales, Sydney, Australia
\end{abstract}

Correspondence to Jeeva Kanesarajah, Public Health Building, School of Public Health, The University of Queensland, Brisbane, QLD

4006, Australia

j.kanesarajah@uq.edu.au

Received 7 June 2015 Revised 10 September 2015 Accepted 10 October 2015 Published Online First 13 November 2015

\section{CrossMark}

To cite: Kanesarajah J, Waller $\mathrm{M}$, Zheng WY, et al. J R Army Med Corps 2016;162:366-372.

\section{ABSTRACT}

Introduction Unit cohesion has been shown to bolster the mental health of military personnel; hence, it is important to identify the characteristics that are associated with low unit cohesion, so that interventions to improve unit cohesion can be targeted and implemented. Little is known about the factors associated with low unit cohesion. This research aims to identify demographic, military service and deployment factors associated with low unit cohesion.

Methods Data from a self-reported cross-sectional study of 11411 current or ex-serving Australian military personnel deployed to Iraq or Afghanistan between 2001 and 2009 were used. Multivariable logistic regression was used to investigate the research aims.

Results Being female (adjusted OR (aOR) $(95 \% \mathrm{Cl})$ 1.35 (1.21 to 1.51)), non-commissioned officer (aOR $(95 \% \mathrm{Cl}) 1.50$ (1.39 to 1.62$)$ ), lower ranked (aOR (95\% CI) $1.74(1.51$ to 2.01$))$ or having left military service (aOR $(95 \%$ CI) 1.71 (1.46 to 2.02)) was associated with reporting low unit cohesion. Potentially modifiable factors such as performing logistic roles on deployment (aOR $(95 \% \mathrm{Cl}) 1.13(1.01$ to 1.27$))$, dissatisfaction with work experience on deployment such as working with colleagues who did not do what was expected of them (aOR (95\% Cl) 4.09 (3.61 to 4.64)), and major problems at home while deployed (aOR $(95 \%$ Cl) 1.50 (1.38 to 1.63)) were also associated with reporting low unit cohesion.

Conclusions This is the first study to identify demographic, military service and deployment factors associated with low unit cohesion. The modifiable nature of unit cohesion means that military leaders could use this information to identify subgroups for targeted resilience interventions that may reduce vulnerabilities to mental health problems and improve the job satisfaction, preparedness and deployment experiences of serving members.

\section{INTRODUCTION}

Unit cohesion is the level of commitment and support that unites military personnel in a unit. There is evidence that unit cohesion improves performance and operational effectiveness ${ }^{1}$ and is associated with greater well-being, greater job satisfaction, superior unit performance and lower incidence of disciplinary problems. ${ }^{2}$ Previous research has demonstrated the utility of unit cohesion as a resilience tool. ${ }^{3}$ It reduces vulnerability to mental health problems such as post-traumatic stress disorder (PTSD), ${ }^{4-7}$ common mental disorder, ${ }^{5} 6$ depression $^{8}$ and physical ill health. ${ }^{5}$ In some

\section{Key messages}

- This is the first study to identify demographic, military service and deployment factors associated with low unit cohesion.

- Potentially modifiable deployment factors such as working with colleagues who did not do what was expected of them on a very difficult experience on deployment, and having to do work on deployment that did not match the military personnel's trade experience and ability were strongly associated with low unit cohesion.

- These findings could be used by the military for targeted resilience interventions, which may reduce the military personnel's vulnerability to mental health, and to improve the job satisfaction, preparedness, unit cohesion and deployment experiences of its serving members as well as assessing members' suitability for deployment.

studies, heavy alcohol use has been associated with poor unit leadership ${ }^{9}$ and higher level of comradeship. 59

Little is known about the demographic, socioeconomic and deployment factors associated with unit cohesion. The few previous studies have focused solely on demographic factors and their associations with unit cohesion, and the authors suggest that unit cohesion is not meaningfully related to demographic characteristics. ${ }^{4} 10$ Nevertheless, it is important to identify potentially modifiable factors associated with low unit cohesion so that strategies can be developed and implemented to improve unit cohesion. Equally, it is important to identify characteristics of groups of people who may benefit from targeted interventions to improve their resilience against mental health vulnerabilities. This study aims to identify subgroups at risk of low unit cohesion by examining demographic, military service and deployment-related characteristics in a cohort of Australian military veterans deployed to the Middle East Area of Operations (MEAO) between 2001 and 2009.

\section{METHODS}

Study design and participants

This research used data from a self-reported, crosssectional, retrospective survey of current and 
ex-serving members of the Australian Defence Force (ADF) who deployed to the MEAO between 1 October 2001 and 31 December 2009. This study included MEAO veterans deployed to Iraq, Afghanistan or supporting operations in these locations (including ships in the Persian Gulf) during this period.

Data were collected in 2010 and 2011 through online surveys or hard copy questionnaires and included questions on deployment history, current health and deployment experiences. A total of 26239 eligible ADF members were invited to participate, and 14032 (53\%) responded to the survey. Analysis of non-response showed that response rates were significantly higher among females, older ADF members, those in the Air Force, officers or those still actively serving in the military. ${ }^{11}$ Participation was voluntary and informed consent was obtained prior to participation.

\section{Unit cohesion}

Participants retrospectively answered questions on their perception of unit cohesion during their most recent deployment to the MEAO. These items were adapted from the US Deployment Experiences Survey with some variation to the questions. These items have also been used in other military studies. ${ }^{12} 13$ Participants were asked to rate their strength of agreement to each item (5: strongly agree; 4: agree; 3 : neither agree nor disagree; 2 : disagree; 1 : strongly disagree).

The unit cohesion items were:

- I felt a sense of comradeship (or closeness) between myself and other people in my unit.

- There was someone I could go to in my unit if I had a personal problem.

- My superiors were interested in what I did or thought.

- I felt well informed about what was going on in my unit.

- I had good communication with other Australian forces/ Australian HQ from my unit.

When the unit cohesion items were assessed for internal consistency and unidimensionality, there was good evidence that they measured a single construct, with a high degree of interrelatedness (Cronbach's $\alpha=0.84) .{ }^{14}$ Furthermore, each item was found to be at least moderately positively correlated to the remaining items $(r \geq 0.36, p<0.001)$, thus providing a rationale to combine the unit cohesion items into a single scale.

To create a unit cohesion scale, response scores to each item were summed to form a total score. Total scores range from 5 to 25 where lower scores indicate poorer unit cohesion. The distribution of scores was skewed and had a strong ceiling effect (14.9\% of respondents had the maximum score of 25). Therefore, participants were categorised into one of three unit cohesion levels, high (total score 23-25), medium (total scores 18-22) and low (total scores 5-17) based on the shape of the distribution of scores to ensure an adequate number of participants in each category for analysis purposes (Figure 1).

\section{Demographic, military service and deployment variables}

Demographic and military service variables used in this study included gender, age (18-29, 30-39, 40+ years), relationship status, service (Navy, Army, Air Force), service status (regulars, active reserves, inactive reserves, ex-serving), rank and number of times deployed to the MEAO (once, twice, three or more) at survey completion. Variables relating to participants' most recent MEAO deployment included location of deployment (Iraq or Afghanistan), whether they deployed as a reservist, whether they deployed with their parent unit and their role on their most recent deployment to the MEAO.
Variables relating to perception of work carried out during deployment were assessed. These included whether ADF members perceived that their work in theatre matched their trade experience and ability, whether their colleagues did what was expected of them on one very difficult experience and their reports of any major problems at home while on deployment. Participants' perceptions of pressure to deploy and treatment by host unit (for those who did not deploy with their parent unit) were also investigated.

\section{Statistical analysis}

All analyses were carried out using SAS V.9.3 for Windows. To account for the non-response bias, the data were weighted for non-response based on the final response rates in the three services (Navy, Army, Air Force), four service status groups (active regulars, active reserves, inactive reserve and ex-serving), three rank categories (commissioned officers, non-commissioned officers, lower ranks) and among males and females. This resulted in 72 strata, and weights calculated were the inverse of the response rate in each stratum. Unless otherwise stated, the frequencies, medians and quartiles reported are unweighted but other summary statistics presented, such as percentages, ORs, adjusted ORs (aORs) and 95\% CIs, were weighted for non-response.

The estimated unweighted medians and quartiles reported were calculated for those who responded to all items in each measure of interest. In the categorisation of the unit cohesion levels, if someone did not complete all items in the unit cohesion scale but it was still possible to definitively determine their category based on their available responses, that person was assigned to that category.

To determine which factors were associated with low unit cohesion, the level of unit cohesion variable was dichotomised to (1) low unit cohesion and (0) combined medium and high groups. In the unadjusted model, binary logistic regression was used to model the association between low unit cohesion and each demographic, military service and deployment variables.

Adjusted binary logistic regression was carried out separately for demographic variables, general deployment experiences variables, and work and relationship-related deployment experiences variables. In the adjusted models, only variables that were statistically significantly associated with low unit cohesion at the $5 \%$ level in the unadjusted analysis were included in the model.

\section{RESULTS}

A total of $11555 \mathrm{ADF}$ members provided responses to at least one unit cohesion item, and 11411 participants provided sufficient responses to be assigned a unit cohesion level. Overall, the majority of participants responded favourably to each unit cohesion item (Figure 2) with $86 \%$ of participants reporting they felt a sense of comradeship with unit members. At survey completion, the median time since participants had last deployed to the MEAO was 3 years.

Around $25 \%$ of participants reported low unit cohesion (Table 1). Female participants, non-commissioned officers, lower ranks and those who were reservists or ex-serving ADF members when they completed the survey were more likely to recall low unit cohesion during their most recent deployment to the MEAO. Those who 'did not know anyone they deployed with on their most recent deployment' or performed logistic roles on deployment were also more likely to report low unit cohesion (Table 2). Those who deployed as reservist to the MEAO were just as likely to report low unit cohesion as those who deployed as regulars. 
Figure 1 Distribution of unit cohesion total scores.

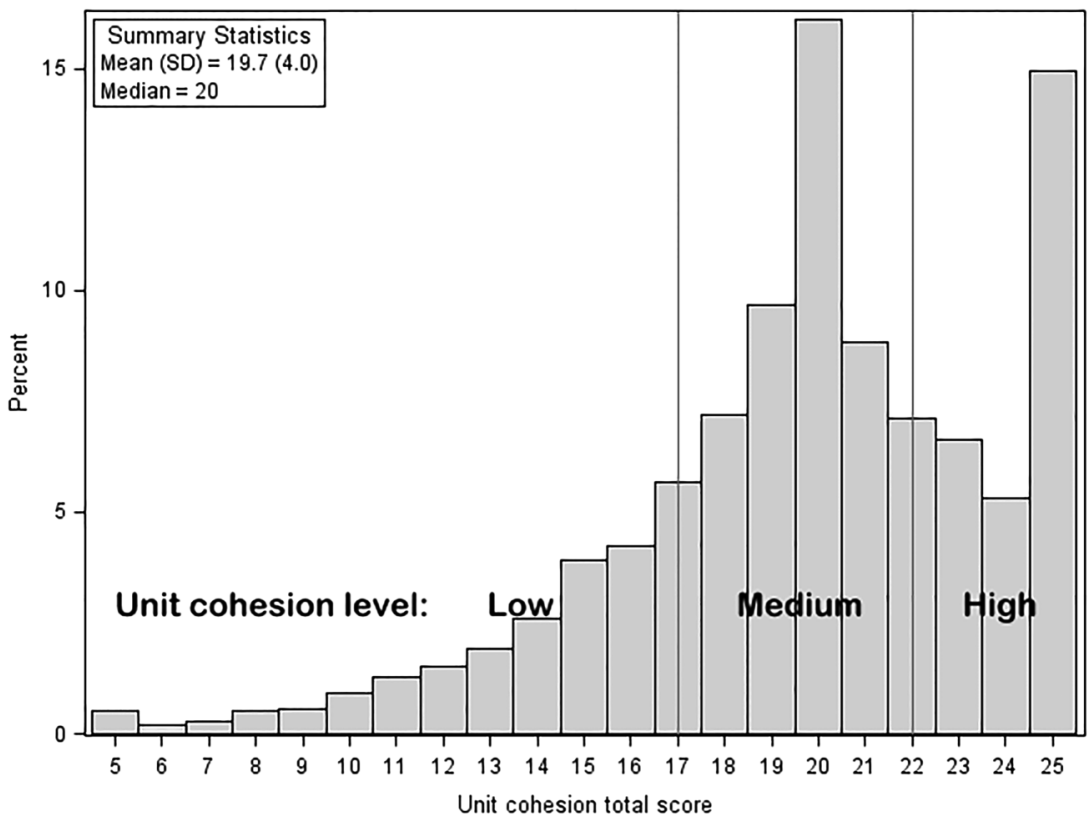

Low unit cohesion was strongly associated with reporting adverse work experiences on deployment including having to do work on deployment that was above or beneath the ADF member's ability (Table 3). Those who 'worked with colleagues who did not do what was expected of them on one very difficult experience on deployment' were considerably more likely to report low unit cohesion than those whose colleagues did what was expected of them (aOR (95\% CI) 4.09 (3.61 to 4.64)). Those who experienced major problems at home while deployed were slightly but significantly more likely to report low unit cohesion (aOR (95\% CI) 1.50 (1.38 to 1.63)). For completeness, results on the association between low unit cohesion and age, relationship status, service, number of deployments to the MEAO and location of deployment are presented online in Supplementary Table 1

\section{Participants' perceptions of pressure to deploy and} treatment from host unit

Approximately $91 \%$ of participants did not feel pressure from their unit to volunteer for the deployment (data not shown); of these, $21 \%$ reported low unit cohesion. In contrast, $42 \%$ of those who felt pressure to deploy, whether from the chain of command or from members in their unit, reported low unit cohesion.

Approximately $82 \%$ of participants who did not deploy with their parent unit felt they were treated equally or better by members of their host unit (data not shown); one-fifth of these participants reported low unit cohesion on deployment. In contrast, $57 \%$ of those who felt they were treated worse by members of their host unit reported low levels of unit cohesion.
Figure 2 Australian Defence Force members' responses to unit cohesion items in per cent $(\mathrm{N}=11555)$.

Percentages weighted for non-response; percentages may not sum to 100 due to rounding.

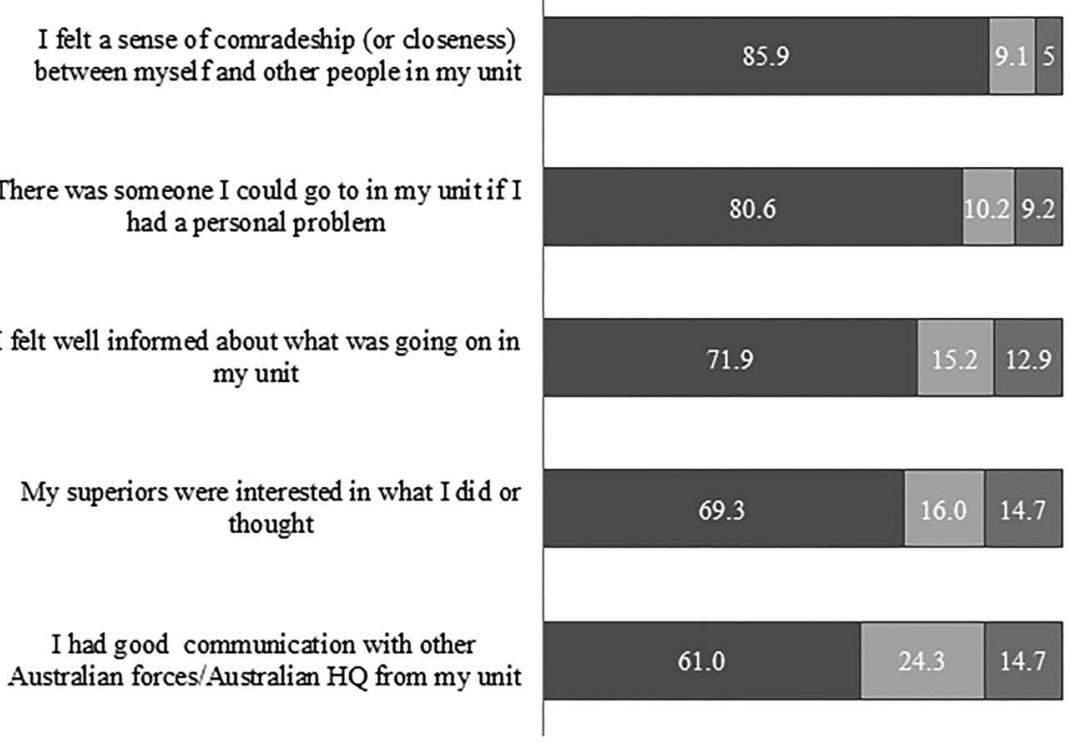

$\square$ Strongly Agree/ Agree $\square$ Neither Agree Nor Disagree $\square$ Strongly Disagree/ Disagree 
Table 1 Associations between level of unit cohesion and demographic and military service characteristics when the survey was completed by Australian Defence Force personnel deployed to the Middle East Area of Operations (MEAO) between 2001 and 2009 ( $N=11411$ ): percentages, unadjusted and adjusted ORs (aORs)

\begin{tabular}{|c|c|c|c|c|c|c|}
\hline & \multicolumn{4}{|c|}{ Unit cohesion, row \%* } & \multicolumn{2}{|c|}{ Low vs medium/high unit cohesion } \\
\hline & $\mathrm{Nt}$ & Low (5-17) & Medium (18-22) & High (23-25) & OR $(95 \% \mathrm{Cl})$ & aOR $(95 \% \mathrm{Cl}) \ddagger$ \\
\hline Overall & 11411 & 25.3 & 48.6 & 26.1 & & \\
\hline \multicolumn{7}{|l|}{ Gender } \\
\hline Male & 10074 & 24.8 & 48.9 & 26.3 & 1 (ref) & 1 (ref) \\
\hline Female & 1337 & 29.7 & 46.0 & 24.3 & $1.28(1.16$ to 1.42$)$ & $1.35(1.21$ to 1.51$)$ \\
\hline \multicolumn{7}{|c|}{ Service status at survey completion } \\
\hline Regulars & 8294 & 22.9 & 49.4 & 27.7 & 1 (ref) & 1 (ref) \\
\hline Active reserves & 1371 & 27.5 & 47.3 & 25.2 & $1.28(1.15$ to 1.42$)$ & 1.29 (1.16 to 1.44$)$ \\
\hline Inactive reserve & 1132 & 28.2 & 48.0 & 23.8 & 1.32 (1.18 to 1.48$)$ & 1.24 (1.10 to 1.41$)$ \\
\hline Ex-serving & 614 & 37.2 & 44.5 & 18.3 & 1.99 (1.71 to 2.33$)$ & 1.71 (1.46 to 2.02 ) \\
\hline \multicolumn{7}{|l|}{ Rank } \\
\hline Commissioned officer & 3527 & 19.1 & 50.1 & 30.8 & 1 (ref) & 1 (ref) \\
\hline Non-commissioned officer & 6692 & 26.4 & 48.1 & 25.5 & 1.52 (1.41 to 1.64$)$ & 1.50 (1.39 to 1.62$)$ \\
\hline Lower rank & 1192 & 31.7 & 47.7 & 20.6 & 1.97 (1.73 to 2.23$)$ & $1.74(1.51$ to 2.01$)$ \\
\hline
\end{tabular}

*Percentages weighted for non-response.

tUnweighted totals,

¥Only variables statistically significantly associated with low unit cohesion at the $5 \%$ level in the unadjusted analysis were included in the model. Adjusted models include gender, age group, service, service status, rank and number of deployments to the MEAO variables at survey completion.

\section{DISCUSSION}

The few studies that have directly investigated factors associated with unit cohesion have primarily focused on demographics factors alone. $^{4} 10$ To date, there has been no direct evidence of any characteristics consistently related to unit cohesion. This study identified several demographic and military service factors associated with the reporting of low unit cohesion, such as being female, non-commissioned officer, lower rank or no longer actively serving in the military. Low unit cohesion was also associated with potentially modifiable deployment factors including experiencing major problems at home while deployed, having to do work on deployment that did not match their ability, working with colleagues who did not perform as expected and marginally associated with performing logistics roles on deployment. Given the strong association with mental health, ${ }^{4-6} 8$ military personnel reporting low unit cohesion may be at greater risk of mental health problems.

Table 2 Associations between level of unit cohesion and deployment experiences in the most recent Middle East Area of Operations (MEAO) deployment, among Australian Defence Force personnel deployed to the MEAO between 2001 and 2009 ( $N=11$ 411): percentages, unadjusted and adjusted ORs (aORs)

\begin{tabular}{|c|c|c|c|c|c|c|}
\hline & \multirow[b]{2}{*}{$\mathrm{N} \dagger$} & \multicolumn{3}{|c|}{ Unit cohesion, row \%* } & \multicolumn{2}{|c|}{ Low vs medium/high unit cohesion } \\
\hline & & Low (5-17) & Medium (18-22) & High (23-25) & OR $(95 \% \mathrm{Cl})$ & aOR $(95 \% \mathrm{Cl}) \ddagger$ \\
\hline \multicolumn{7}{|l|}{ Service status on deployment } \\
\hline Full-time member & 10830 & 25.3 & 48.8 & 25.9 & 1 (ref) & \\
\hline Reservist & 506 & 26.5 & 45.0 & 28.5 & 1.07 (0.91 to 1.25$)$ & \\
\hline \multicolumn{7}{|l|}{ Deployed with parent unit? } \\
\hline Yes & 5504 & 23.9 & 48.9 & 27.2 & 1 (ref) & 1 (ref) \\
\hline No, but deployed with some unit members & 3114 & 26.0 & 49.0 & 25.0 & 1.12 (1.03 to 1.22$)$ & $1.24(1.13$ to 1.36$)$ \\
\hline No, did not deployed with anyone known & 2327 & 28.2 & 47.5 & 24.3 & 1.25 (1.15 to 1.37$)$ & 1.60 (1.45 to 1.78$)$ \\
\hline NA, did not have a parent unit & 441 & 25.5 & 46.2 & 28.3 & 1.09 (0.90 to 1.32 ) & $1.36(1.11$ to 1.67$)$ \\
\hline \multicolumn{7}{|l|}{ Main role on deployment } \\
\hline Combat & 3613 & 25.1 & 47.8 & 27.1 & 1 (ref) & 1 (ref) \\
\hline Combat support & 2323 & 25.1 & 49.7 & 25.2 & 1.00 (0.90 to 1.11$)$ & 0.96 (0.86 to 1.07$)$ \\
\hline Medical/welfare & 479 & 27.9 & 45.8 & 26.4 & 1.15 (0.97 to 1.37$)$ & 1.20 (1.00 to 1.46$)$ \\
\hline Air crew & 688 & 19.3 & 51.7 & 29.1 & 0.71 (0.61 to 0.83 ) & $0.95(0.79$ to 1.14$)$ \\
\hline Maritime operations & 286 & 30.3 & 50.9 & 18.8 & 1.30 (1.02 to 1.65$)$ & 1.30 (1.00 to 1.68$)$ \\
\hline Logistics & 2356 & 27.6 & 48.6 & 23.8 & 1.14 (1.03 to 1.27$)$ & 1.13 (1.01 to 1.27$)$ \\
\hline Administrative & 221 & 24.8 & 52.5 & 22.7 & 0.98 (0.78 to 1.25$)$ & $0.94(0.72$ to 1.23$)$ \\
\hline Other roles & 1258 & 23.1 & 47.7 & 29.2 & 0.90 (0.80 to 1.01$)$ & $1.04(0.91$ to 1.19$)$ \\
\hline
\end{tabular}

${ }^{*}$ Percentages weighted for non-response; Percentages may not sum to 100 due to rounding.

tUnweighted totals; totals may not add up due to missing responses.

¥Only variables statistically significantly associated with low unit cohesion at the $5 \%$ level in the unadjusted analysis were included in the model. Adjusted regression model includes deployed with parent unit, main role in theatre variables, and adjusted for gender, age group, service, service status (at survey completion), rank and number of deployments to the MEAO at survey completion. 
Table 3 Associations between level of unit cohesion and perceptions of work carried out during deployment and relationship with family while deployed, among Australian Defence Force personnel deployed to the Middle East Area of Operations (MEA0) between 2001 and 2009 ( $\mathrm{N}=11$ 411): percentages, unadjusted and adjusted ORs (aORs)

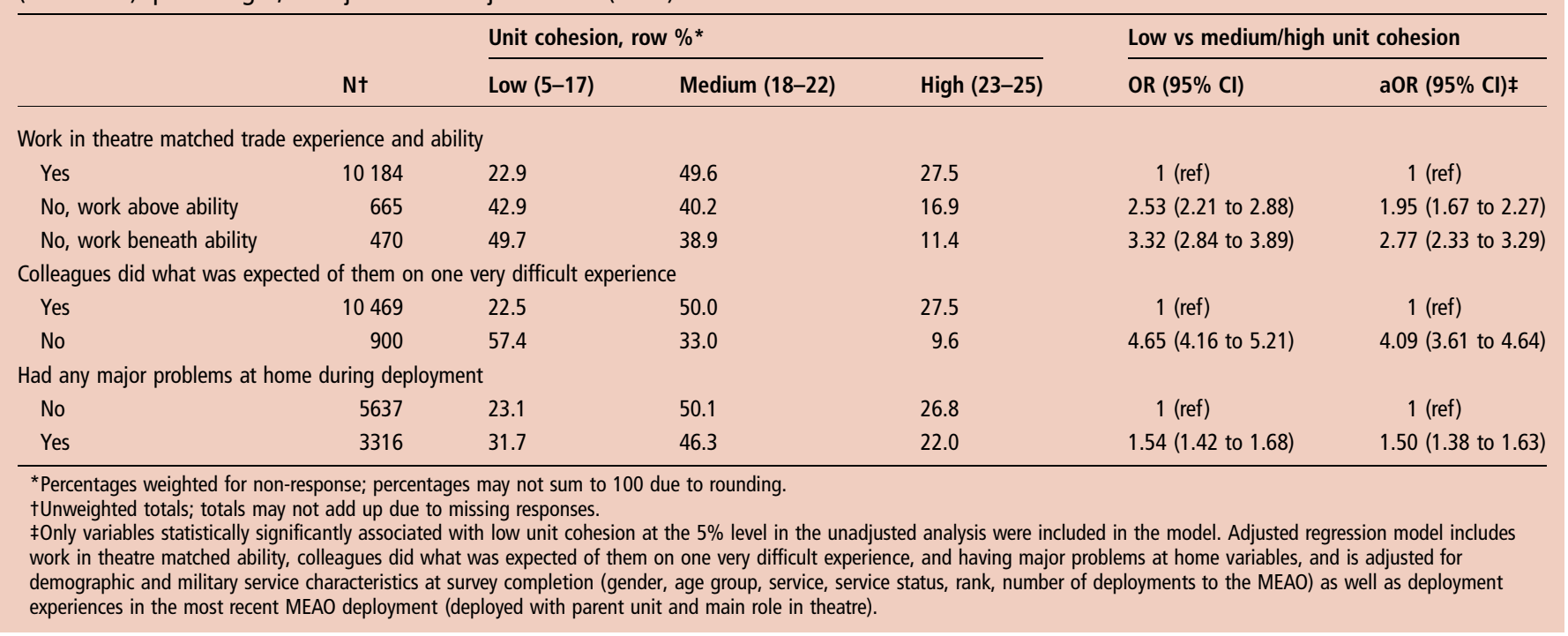

In the interpretation of these results, the cross-sectional, retrospective nature of the study means that causation cannot be inferred. Recall bias and reverse causation are possible, that is, those with poor mental health may be more likely to reflect poorly on their deployment experiences, leading to perceptions of low unit cohesion.

Despite these limitations, previous research supports our finding that female ADF members are slightly, but statistically significantly, more likely to report low unit cohesion compared with male ADF members. ${ }^{15}{ }^{16}$ Female military personnel, particularly those exposed to combat on deployment, may be more vulnerable to mental health problems such as PTSD ${ }^{17} 18$ and depression. ${ }^{19}{ }^{20}$ With the recent lifting of the ban by the ADF on women occupying frontline roles, ${ }^{21}$ understanding how deployment-related risk and resilience factors impact on women and their mental health has become increasingly important to mitigate potential mental health problems in this group.

Most research on unit cohesion is based on groups of currently serving military personnel; hence, little is known about the association between unit cohesion and military retention. These findings suggest that the reporting of low unit cohesion was higher among those no longer serving in the military. Ex-serving ADF members may have discharged due to feeling disillusioned from negative deployment experiences such as poor unit cohesion. Conversely, they may have left military service due to other reasons such as poor health and their perceptions of unit cohesion may biased by subsequent negative experiences after deployment.

Our finding that non-commissioned officers or lower ranked ADF members were more likely to report lower unit cohesion on deployment than commissioned officers contrasts with previous research. ${ }^{4}$ This discrepancy between our study and research by Dickstein and colleagues may be attributed to differences in the characteristics of participants (ie, 11511 Australian military personnel from all ranks, in the Army, Navy or Air Force deployed to Iraq or Afghanistan vs 705 US Air Force medical personnel deployed to Iraq). However, our findings are consistent with Whitehall II study of British civil servants, which found that employees in the lower grades of employment were up to six times more likely than those in the higher grades to report low levels of work control or social support at work from colleagues and managers. ${ }^{22}$

To our best knowledge, no prior research have directly investigated the association between role on deployment and unit cohesion. Our findings suggest those deployed on logistic roles (eg, supply, driver, catering, trades) were marginally more likely to report lower unit cohesion than those with combat roles. This may be due to the isolated nature of these jobs, which may result in little interaction with others, thus contributing to low unit cohesion scores. Alternatively, military personnel in these roles may have been force-assigned to provide service support to members of the host unit, hence may be less familiar with members of the host unit.

ADF members who felt their work on deployment did not match their trade experience or ability were more likely to report low unit cohesion. Findings from other research suggest that feelings of unpreparedness, particularly the perception that work on deployment was above one's ability, led to greater risk of PTSD symptoms. ${ }^{12}$ This lack of preparedness may also lead to feelings of loss of control or feeling unable to work independently to complete the task at hand. Alternatively, those who were overprepared may not have felt challenged by their tasks on deployment, leading to boredom and dissatisfaction with their deployment experience.

Deployed military personnel may face dual stressors from experiences in the warzone and on the home front. Research with female veterans suggests that separation from family during deployment is particularly stressful to those with caring responsibilities; ${ }^{23}$ they may be expected to fulfil their familial responsibilities on deployment. Our findings shows that ADF members experiencing major problems at home while deployed were more likely subsequently to report low unit cohesion. This may result in the military personnel being preoccupied with problems at home while on deployment, which may impact on their performance in theatre as well as contribute to perceptions of lack of closeness with their unit members.

Those deployed as reservists to the MEAO were as likely to report low unit cohesion as those deployed as regulars, contrasting with work by Browne and colleagues, who found that deployed reserve units reported slightly lower unit cohesion and 
effective leadership compared with deployed regulars. ${ }^{24}$ Those authors suggested that differences in unit cohesion between the deployed reservists and regulars partly contributed to negative health effects in reservists. The difference between the two findings may be attributed to differences in study design, namely the oversampling of reservists in the study by Brown et al. With only about $5 \%$ of veterans in this study deployed as reservists, the sample size in our study may not be large enough to be able to detect meaningful differences between those deployed as reservists and those deployed as regulars.

\section{Strength and limitations}

The study used data from a large cohort of current and ex-serving ADF members deployed to the MEAO, and the nature of the military operations indicates that the findings may be generalisable to other contemporary military populations. The large sample size generally gives good statistical power, and the results were weighted to account for possible non-response bias.

The cross-sectional nature of the survey means that causation cannot be inferred as both the exposure and outcome were reported at the same time; participants may also have been influenced by the retrospective nature of the study. Some respondents may have recalled their perception of unit cohesion and other deployment experiences up to 10 years after deploying to the Middle East. Their recall may have been influenced by their mood while completing the survey, their postwar experiences, as well as any current mental health issues. On the other hand, this long lead time could also allow veterans to come to terms with any negative experiences on deployment that might have influenced their perceptions of unit cohesion.

The unit cohesion scale has not been validated but it was found to have good internal reliability in this cohort of ADF members. As there are no existing cut points for unit cohesion score in the literature, the score was categorised into three groups based on the shape of the distribution of scores. However, the distribution of unit cohesion scores may vary across cohorts, so these cut points may not be applicable to other studies, making comparisons across studies difficult.

\section{Implications}

The potentially modifiable nature of unit cohesion means that information on characteristics associated with low unit cohesion could be used by military leaders and unit commanders to identify groups for targeted interventions such as resilience training, which may, in turn, reduce the military personnel's vulnerability to mental health problems. ${ }^{25}$ Given the strong association between low unit cohesion and poor mental health, this knowledge may further assist in assessing members' suitability for deployment and identify those at risk of health issues post deployment for targeted interventions. Where possible, military leadership should ensure unit members understand their role on deployment, are prepared for work on deployment and perform as expected. If not, adequate training and support should be made available to them on deployment.

\section{CONCLUSION}

This is the first study to have identified key military workforce characteristics associated with low unit cohesion. These findings could be used by the military to improve the unit cohesion, preparedness and deployment experiences of its workforce, as well as to identify subgroups for targeted resilience interventions.
Acknowledgements The authors thank the veterans who participated in the survey and the Australian Department of Defence for funding the MEAO Census Study and facilitating the data collection.

Contributors $J K$ contributed to the conception of the research aims, performed the statistical analysis, review of the literature, interpreted the data and drafted all sections of the article. MW contributed to the analysis and interpretation of the data, and provided critical intellectual comment to revise the drafts. WYZ contributed to the conception of the research aims, interpretation of the data, and provided critical intellectual comment to revise the drafts. AJD contributed to the analysis and interpretation of the data, and provided critical intellectual comment to revise the drafts. All authors approved of the version of the article submitted for consideration.

Funding The Australian Government Department of Defence (http://www.defence. gov.au/) funded and facilitated data collection of the Middle East Area of Operations (MEAO) Census Study, which was completed in 2012. The authors made use of data from the MEAO Census Study for this research. However, this research was completed in the course of their employment-therefore, the authors received no specific funding from the Australian Department of Defence for this work.

Disclaimer The opinions or assertions contained herein are the private views of the authors and are not to be construed as official or representing the views of the Australian Defence Force or Department of Defence.

Competing interests None declared.

Ethics approval Australian Department of Defence Joint Health Command Low Risk Ethical Review Panel \& The University of Queensland Behavioural and Social Sciences Ethical Review Committee.

Provenance and peer review Not commissioned; externally peer reviewed.

Data sharing statement The raw data may be made available for reuse subject to ethical approval by Australian Department of Defence Joint Health Command Low Risk Ethical Review Panel and full data access approval by the Military and Veterans' Health Research Data Access Committee (MVHRDAC).

Open Access This is an Open Access article distributed in accordance with the Creative Commons Attribution Non Commercial (CC BY-NC 4.0) license, which permits others to distribute, remix, adapt, build upon this work non-commercially, and license their derivative works on different terms, provided the original work is properly cited and the use is non-commercial. See: http://creativecommons.org/ licenses/by-nc/4.0/

\section{REFERENCES}

1 Ahronson A, Cameron JE. The nature and consequences of group cohesion in a military sample. Mil Psychol 2007;19:9-25.

2 Oliver LW, Harman J, Hoover E, et al. A quantitative integration of the military cohesion literature. Mil Psychol 1999;11:57-83.

3 MacManus D, Jones N, Wessely S, et al. The mental health of the UK Armed Forces in the 21st century: resilience in the face of adversity. J $R$ Army Med Corps 2014;160:125-30.

4 Dickstein BD, McLean CP, Mintz J, et al. Unit cohesion and PTSD symptom severity in Air Force medical personnel. Mil Med 2010;175:482-6.

5 Du Preez J, Sundin J, Wessely $S$, et al. Unit cohesion and mental health in the UK armed forces. Occup Med (Lond) 2012;62:47-53.

6 Jones N, Seddon R, Fear NT, et al. Leadership, cohesion, morale, and the mental health of UK Armed Forces in Afghanistan. Psychiatry 2012;75:49-59.

7 Mulligan $\mathrm{K}$, Jones N, Woodhead C, et al. Mental health of UK military personnel while on deployment in Iraq. Br J Psychiatry 2010;197:405-10.

8 Armistead-Jehle P, Johnston SL, Wade NG, et al. Posttraumatic Stress in U.S. Marines: The Role of Unit Cohesion and Combat Exposure. J Couns Dev 2011;89:81-8

9 Browne TE, Iversen A, Hull L, et al. How do experiences in Iraq affect alcohol use amongst male UK Armed Forces personnel? J Occup Environ Med 2008:65:628-33.

10 Siebold GL, Lindsay TJ. The relation between demographic descriptors and soldier-perceived cohesion and motivation. Mil Psychol 1999;11:109-28.

11 Dobson A, Treloar S, Zheng WY, et al. The Middle East Area of Operations (MEAO) Health Study. Census Study Report. The University of Queensland, Centre for Military and Veterans' Health, Brisbane, Australia, 2013.

12 Iversen AC, Fear NT, Ehlers A, et al. Risk factors for post-traumatic stress disorder among UK Armed Forces personnel. Psychol Med 2008;38:511-22.

13 Rona RJ, Hooper R, Jones $M$, et al. The contribution of prior psychological symptoms and combat exposure to post Iraq deployment mental health in the UK military. J Trauma Stress 2009;22:11-19.

14 Cronbach LJ. Coefficient alpha and the internal structure of tests. Psychometrika 1951;16:297-334.

15 Carter-Visscher R, Polusny MA, Murdoch M, et al. Predeployment gender differences in stressors and mental health among U.S. National Guard troops poised for Operation Iraqi Freedom deployment. J Trauma Stress 2010;23:78-85. 


\section{Original article}

16 Kline A, Ciccone DS, Weiner M, et al. Gender differences in the risk and protective factors associated with PTSD: a prospective study of National Guard troops deployed to Iraq. Psychiatry 2013;76:256-72.

17 Mota NP, Medved M, Wang J, et al. Stress and mental disorders in female military personnel: comparisons between the sexes in a male dominated profession. J Psychiatr Res 2012:46:159-67.

18 Skopp NA, Reger MA, Reger GM, et al. The role of intimate relationships, appraisals of military service, and gender on the development of posttraumatic stress symptoms following Iraq deployment. I Trauma Stress 2011;24:277-86.

19 Gibbons SW, Hickling EJ, Barnett SD, et al. Gender differences in response to deployment among military healthcare providers in Afghanistan and Iraq. J Womens Health (Larchmt) 2012;21:496-504.

20 Luxton DD, Skopp NA, Maguen S. Gender differences in depression and PTSD symptoms following combat exposure. Depress Anxiety 2010;27:1027-33.
21 Women in the Australian Defence Force. Removal of Gender Restrictions Secondary Removal of Gender Restrictions 27/9/2012, 2012. http://www.defencejobs.gov.au/ women/RemovalofGenderRestrictions/15/

22 North FM, Syme SL, Feeney A, et al. Psychosocial work environment and sickness absence among British civil servants: The Whitehall II Study. Am J Public Health 1996:86:332-40.

23 Feldman S, Hanlon C. Count us in: the experiences of female war, peacemaking, and peacekeeping veterans. Armed Forces Soc 2011;38:205-24.

24 Browne T, Hull L, Horn O, et al. Explanations for the increase in mental health problems in UK reserve forces who have served in Iraq. $\mathrm{Br}$ I Psychiatry 2007;190:484-9.

25 Pietrzak RH, Johnson DC, Goldstein MB, et al. Psychosocial buffers of traumatic stress, depressive symptoms, and psychosocial difficulties in veterans of Operations Enduring Freedom and Iraqi Freedom: the role of resilience, unit support, and postdeployment social support. J Affect Disord 2010;120:188-92. 